\title{
Menopausia: \\ representaciones sociales y prácticas
}

\author{
Blanca Pelcastre-Villafuerte, Dra. en Psic. Soc., (1) Francisco Garrido-Latorre, M.C ., M. en C., (1) \\ Verónica de León-Reyes, M.S.P. ${ }^{(1)}$
}

\section{Pelcastre-Villafuerte B, Garrido-Latorre F.} Menopausia: representaciones sociales y prácticas Salud Publica Mex 2001:43:408-414. El texto completo en inglés de este artículo está disponible en: http://www.insp.mx/salud/index.html

\section{Resumen}

Objetivo. Caracterizar los significados que se atribuyen al proceso de la menopausia como una primera aproximación al mundo representacional de este evento. Material y métodos. Estudio cualitativo, hecho entre septiembre y octubre de 1998 con 20 mujeres de entre 45 y 65 años de edad, residentes en los municipios de Cuernavaca y Emiliano Z apata del estado de Morelos, México. Se efectuaron sendas entrevistas a profundidad, utilizando una guía con los siguientes apartados: datos sociodemográficos, diagnóstico, sentimientos y sensaciones, cambios en el estilo de vida, cambios fisio ló gicos e información. Resultados Se halla una representación que se construye alrededor de la menopausia en términos de cese de la fertilidad y entrada al envejecimiento. Esta caracterización tiene que ver también con otras construcciones como la concepción de la mujer, del cuerpo y de la sexualidad. Conclusiones. En este artículo se retoman principalmente los resultados con relación a las prácticas de las mujeres como consecuencia de la vivencia que tienen de la menopausia, entre las que sobresale la visita al médico. Se discuten las implicaciones desde el marco de la construcción social de los significados. El texto completo en inglés de este artículo está disponible en: http://www.insp.mx/salud/index.html

Palabras clave: menopausia; representación social; significados; prácticas; México

\author{
Pelcastre-Villafuerte B, Garrido-Latorre F. \\ Social representations \\ and practices of menopause. \\ Salud Publica Mex 2001:43:408-414. \\ The English version of this paper \\ is available at: http://www.insp.mx/salud/index.html
}

\begin{abstract}
A bstract
Objective. To characterize the meanings attributed to menopause, as a first approximation to the representational world of this event. Material and Methods. A qualitative study was conducted between September and 0 ctuber 1998, in twenty wo men aged 45-65 years, residents of Cuernavaca and Emiliano Z apata, municipalities of Morelos state, Mexico. Twenty in-depth interviews were conducted using a guide, to collect data on socio demo graphic variables, diagnosis, feelings and emotions, changes in life style, and physiological changes. Results Findings show that menopause is represented as the end of fertility and the beginning of old age. Characterization of meno pause is related to womanhood, body, and sexuality representations. Conclusions. This paper analyzes women's practices related to life experiences such as medical visits. Implications of these findings are discussed using the social construction of meanings framework. The English version of this paper is available at: http://www.insp.mx/salud/index.html
\end{abstract}

Key words: menopause; social representations meanings; practices; Mexico

(1) Centro de Investigación en Sistemas de Salud, Instituto N acional de Salud Pública, México.

Fecha de recibido: 30 de agosto de 2000 - Fecha de aprobado: 28 de marzo de 2001

Solicitud de sobretiros: D ra. Blanca Pelcastre Villafuerte. Departamento de diseños y modelos alternativos de atención. Instituto $N$ acional de Salud Pública, Centro de Investigación en Sistemas de Salud, D irección de Salud Comunitaria y Bienestar Social.Avenida Universidad N 0655 , colonia santa María A huacatitlán 62508, Cuernavaca Morelos, México.

Correo electrónico: bpelcast@ insp3.insp.mx 
E n la compleja organización cultural de los grupos sociales, las formas concretas de la vida se componen de signos, símbolos y significados con orientaciones diversas; el mejor ejemplo de un sistema simbólico es sin duda el lenguaje, pues a través de él construimos colectivamente la realidad social en la que vivimos. El lenguaje ha sido distinguido como una característica intrínsecamente humana y esencialmente colectiva; diversos autores lo han edificado como primer y último elemento explicativo de todo lo social; algunos como Billig, ${ }^{1}$ uno de los principales representantes del llamado "giro retórico", han puesto énfasis sobre el carácter dilemático del mismo, y otros como Edwards ${ }^{2}$ y Fernández ${ }^{3}$ han trabajado más bien su dimensión metafórica y afectiva, respectivamente, pero sus diversas propuestas coinciden en el punto que el mismo Wittgenstein ${ }^{4}$ sugería desde años antes, la noción de que "...imaginar un lenguaje es imaginar una forma de vida...", resaltando con ello su carácter fundamentalmente creativo. Estos autores, desde diferentes ámbitos y perspectivas, han fortalecido la propuesta de que el discurso es la base de la intersubjetividad y constructor de la realidad social; idea esta última a la cual nos adherimos en el marco explicativo de las representaciones sociales.

El entendimiento y la comunicación en un grupo social, son procesos generados por el carácter básicamente compartido del universo simbólico-imaginativo de sus miembros; en este sentido, el estudio de las representaciones sociales nos permite comprender mejor algunos de los mecanismos involucrados en el proceso de transmisión cultural de este universo.

Denise Jodelet, una de las principales autoras que han desarrollado el concepto de representación social, se ha referido a este proceso como "una forma de pensamiento social", compartido y de carácter práctico, que permite interpretar acontecimientos de la vida diaria, información y características del medio ambiente, tanto como a los otros actores sociales involucrados en esa elaboración. ${ }^{5}$

Las representaciones sociales condensan historia, relaciones sociales, prácticas políticas y prejuicios; ${ }^{6,7,8}$ en este sentido son cambiantes y dependientes del contexto de vida. Los factores que influyen en su conformación se relacionan con las diversas fuentes de información accesibles, y con los diferentes discursos o narrativas circulantes en la atmósfera social.

No identificamos la representación social como un proceso de reconstrucción individual, pues consideramos las características propias que definen su carácter social. Para Ibáñez, ${ }^{9}$ este carácter obedece fundamentalmente a su función: formación de comportamientos y orientación de las comunicaciones sociales. Las rela- ciones sociales dan sentido y significado a las representaciones y viceversa; en este proceso dinámico se construye e interpreta la realidad.

En términos teóricos el concepto de representación social tiene una gran potencialidad en tanto que permite analizar la intersección entre lo psicológico y lo social, es decir, aporta elementos para entender la difícil y a veces desentrañable relación existente entre el pensamiento y el comportamiento social. ${ }^{5}$

Adentrarse en el estudio de las representaciones sociales implica trastocar diferentes ámbitos que funcionan de manera interdependiente: el lenguaje, el pensamiento y el comportamiento ya que, por ejemplo, el significado y las normas implícitas en las prácticas sociales no se encuentran en la mente de los actores, sino fuera de ella, en las prácticas mismas, prácticas que de acuerdo a Shotter ${ }^{10}$ no pueden ser concebidas como un conjunto de acciones individuales, sino esencialmente como modos de relación social o de acción mutua. Nuestras formas de actuar, siguiendo a este autor, se articulan con las condiciones sociales, generadas al mismo tiempo por la interacción establecida a partir de estas formas; sin embargo, se encuentran también estrechamente vinculadas a nuestras visiones de mundo, y así nuestra forma de interpretar la realidad y nuestro contexto de vida, conducen conjuntamente nuestras acciones.

Entre los diversos enfoques sobre las representaciones sociales, ${ }^{*}$ aquí se retoma aquel que considera la representación como una práctica discursiva. Gadamer reconoce que "...en la conversación nos trasladamos constantemente al mundo representativo del otro...", ${ }^{11}$ por tanto el lenguaje interviene efectivamente como organizador de contenido y operador de sentido, por ello Jodelet ${ }^{5}$ remarca que las producciones discursivas de las personas se presentan como el único material mediante el cual se pueden conocer sus representaciones sociales.

El reconocimiento de las funciones del lenguaje y su importancia en la construcción de las realidades sociales, nos permite generar un marco de interpretación de los diferentes discursos de las mujeres en relación con la menopausia y las distintas prácticas derivadas de ellos.

En términos de la presente investigación, los discursos de las informantes se tomaron como punto de partida para reconstruir una primera aproximación a su representación social de la menopausia y establecer posibles nexos explicativos con sus prácticas o acciones.

* Referencia 5. 
El climaterio es, en sentido amplio, un evento biográfico cuya construcción se basa en las diversas opiniones, en las tradiciones populares, en el discurso del proceso desde las ciencias biológicas y desde las sociales. En este sentido debe ser abordado desde una perspectiva integradora, como lo señala Lolas. ${ }^{12}$ En México ha habido pocos intentos por analizar actitudes y prácticas de mujeres en esta etapa de la vida, en una perspectiva más general; en América Latina hay pocos investigadores estudiosos de la sexualidad y del envejecimiento en mujeres. ${ }^{13}$

De acuerdo con la literatura, podemos hablar de diversos puntos de vista en relación con la menopausia. Algunos médicos tienden a verla como una "enfermedad deficitaria" o una patología biológica que requiere atención especializada. Entre ellos se encuentran los teóricos evolucionistas quienes comparten la noción de ser la menopausia un "apéndice" hormonal de la mujer. ${ }^{14,15}$ Otro punto de vista la considera un proceso natural asociado con la edad, y puede transcurrir sin mayores complicaciones.

Es posible identificar un tercer tipo de representación perfilado hacia lo mágico-religioso, combinado con lo natural; en este caso es común el uso regional de términos específicos para referirse a ella, términos que reflejan la cosmovisión de la comunidad. ${ }^{15}$

La investigación de la dimensión social y psicológica de la salud deviene necesidad cuando se tratan de establecer las prioridades en este ámbito, pues una noción amplia de este concepto incluye todas aquellas representaciones que pueden repercutir sobre la percepción del propio cuerpo y el autocuidado. Como primera aproximación a este gran campo de lo simbólico, el presente estudio exploratorio pretendió caracterizar los significados atribuidos al proceso de la menopausia en mujeres que viven en distintas zonas del estado de Morelos, describir sus prácticas y hablar de las acciones que llevan a cabo durante esta etapa de sus vidas.

El acercamiento a la dimensión social de este proceso, potencialmente permite aportar elementos que pueden ser útiles en la planeación de servicios y en cualquier propuesta de mejoramiento sustancial de la atención basada en las necesidades de las usuarias.

\section{Material y métodos}

Para esta exploración de la experiencia representacional de la menopausia, se propuso una aproximación cualitativa para conocer qué piensan algunas mujeres respecto a tal evento. Se consideró la entrevista individual como la técnica metodológica más adecuada para la obtención de esta información, ${ }^{16}$ pues tradi- cionalmente la menopausia se considera un aspecto íntimo correspondiente a la vida privada de las mujeres, y en este sentido, no resulta fácil hablar de ello en grandes grupos. Se podría decir que la menopausia constituye, junto con las enfermedades psicosomáticas y la depresión, lo que Salamonovitz llamara "enfermedades del silencio" ${ }^{17}$

La entrevista individual, en tanto que confidencial, permitió ahondar en temas específicos en relación con los objetivos del estudio.

Se eligieron los municipios de Cuernavaca y Emiliano Zapata para llevar a cabo el estudio. En total se aplicaron 20 entrevistas a profundidad cuyo número final fue determinado bajo el criterio de saturación teórica. ${ }^{18}$ Las colonias visitadas en cada municipio fueron elegidas al azar, y esto tuvo lugar durante los meses de septiembre y octubre de 1998. Las entrevistas fueron grabadas y posteriormente transcritas, y estuvieron basadas en una guía con una sección de datos sociodemográficos como edad, último año de estudios, edad de la menarca, estado civil y número de hijos; además se incluyó el apartado "diagnóstico" cuyo fin fue explorar los procesos o síntomas reconocidos por las mujeres como propios de la menopausia. Para dar cuenta del aspecto afectivo de este proceso, se diseñó el apartado de "sentimientos y sensaciones"; el apartado "cambios en el estilo de vida" exploró todos aquellos aspectos de la vida personal, de pareja y/ o familiar relacionados con el proceso de la menopausia. Se incluyó una sección de "cambios fisiológicos", para abordar los aspectos físicos relacionados con el evento, y una de "información" para saber cuánto y cuál tipo de conocimiento tenían al respecto.

El análisis de la información se hizo siguiendo los pasos propuestos por la Teoría Fundamentada (Grounded Theory), ampliamente probada en el análisis de información cualitativa. ${ }^{19}$

El análisis general incluyó todas las categorías mencionadas, sin embargo, para efectos del presente artículo se retomó únicamente la categoría de "acciones", vinculada a lo que las mujeres hacen para enfrentar la menopausia, por ello, los resultados y la discusión se ven limitados a este aspecto.

\section{Resultados}

\section{Edad}

Mediante el análisis de las entrevistas observamos en las mujeres de entre 40 y 50 años una preocupación y un alivio con relación a la menopausia; la primera está relacionada con síntomas como intensas hemorragias, bochornos y dolores de cabeza referidos por 
otras mujeres de mayor edad cuya experiencia no ha sido muy favorable. El alivio se presenta ante la desaparición del constante temor a quedar embarazadas; aunada a ello aparece también una atribución de orientación negativa relacionada con el envejecimiento, cuya caracterización se basa en ideas como pérdida de energía, achaques e imposibilidad de cumplir con su papel social reproductivo. Aquí ocurre lo que expresara Simone de Beauvoir en su libro Sobre la vejez, "el envejecimiento se caracteriza como un proceso progresivo desfavorable de cambio, ordinariamente ligado al paso del tiempo, que se vuelve perceptible después de la madurez." ${ }^{20}$

En el grupo anterior predomina, por tanto, una ambivalencia definida en el siguiente grupo de edad de 50 a 60 años; en éste se concibe la menopausia como una desesperación, una enfermedad muy fea, un malestar de calorías, cuando das el viejazo, un proceso de pérdida de vida, pérdida de la razón, pérdida de fertilidad, que se vive con calores intensos que salen del cuerpo, según testimonio de las informantes.

Algunas mujeres expresaron también una suerte de liberación del fastidio que significa la menstruación, ello les daba a su vez mayor seguridad en sí mismas.

En cuanto a las relaciones de pareja, expresan que los hombres tienen a sus mujeres sólo para satisfacerles sexualmente, lo cual se convierte en una obligación asumida hacia el marido; en general no comparten la vivencia del proceso menopáusico con sus parejas y manifiestan cómo el deseo sexual desaparece en ellas, adjudicando este hecho a la edad; esto no sucede en los hombres, dicen las mujeres, pues su deseo permanece constante.

Este tipo de afirmaciones habla de una representación particular del ser mujer y la vivencia de la sexualidad en relación con ésta; ello nos sugiere una naturalización de la diada edad-líbido, en donde sexualidad y edad mantienen una relación inversamente proporcional una vez alcanzada la expresión máxima de aquélla.

En contraste con los dos grupos anteriores, en las mujeres de 60 años y más, los mitos y las creencias son ampliamente socorridos en este tema. Se identificaron mitos alusivos a dos aspectos de su persona, un primer aspecto relacionado con su sistema reproductivo, y un segundo concerniente al rol social de las mujeres. En relación con el primer aspecto una mujer expresó:

“... yo soy una persona (...) que yo nunca le pregunté a mi mamá, ¿mamá, por qué esto?, ¿mamá, por qué lo otro?, nunca le pregunté a mi madre. Solamente pues, las amigas que me llegaban a decir algo, ya estaba yo una muchacha como de unos 14 años y todavía no sabía yo qué tenía que hacer la mujer para sus hijos, sí, porque (...) mi mamá cuando tuvo a su tercer hijo, me mandó a un mandado y ya llegué yo, y cuando entré, escuché yo que lloraba mi hermano (...) y yo le pregunté, ¿cómo?, ¿cómo éste resultó ahí?, dice: por la boca”.

Otras mujeres mencionaron algunas recomendaciones como no comer limón o bañarse con agua fría para que no se corte la regla, así como la creencia de que la mente lo controla todo $y$

“...usted podría hasta hacer que a usted le bajara su regla si usted lo piensa así...".

Respecto al rol social de las mujeres, una de ellas expresó, refiriéndose a lo que sintió cuando dejó de bajarle la regla:

“...lo asocias también con tu incapacidad de ser mujer, lo cual es otro de los problemas que modifican tu conducta...".

Probablemente esta incapacidad esté identificada con la función social reproductiva. Otra mujer manifestó, aludiendo al rol que como madres deben desempeñar:

“...siempre hemos vivido así, es como una gallina que está uno cubriendo a sus hijos (...), porque si uno como madre no hace eso por sus hijos, pues no, pienso yo que no es madre...".

No tocarse el cuerpo, ni sentarse con las piernas abiertas, para evitar que ...entre un animal y que dejáramos de ser señoritas..., fue otra idea manifestada por estas mujeres.

La presencia de este tipo de ideas, aunque no muy frecuente en este grupo de mujeres, nos da cuenta de las creencias que se mantienen y que mediante la crianza y la educación transmiten a sus descendientes.

Estas mujeres aluden a una sensación de mutilación cuando se presenta su menopausia, la cual podría evitarse con un buen trato hacia ellas por parte de toda la gente. Esta alusión nos sugiere que la menstruación es un elemento decisivo del significado de ser mujer. Cuando desaparece las mujeres sienten haber perdido una parte de sí mismas, de lo que las define e identifica. 


\section{Acciones}

Quienes no refirieron ninguna acción concreta en relación con la menopausia, aluden a este proceso en términos de un deterioro natural del organismo. Se reportan síntomas de cansancio y dolores, pero éstos no constituyen motivo de consulta pues no se concibe como una enfermedad que requiera atención especializada.

El uso de remedios caseros no es una opción a la que recurran con frecuencia estas mujeres, sin embargo una de ellas habla del uso de algún tipo de remedio sin especificación del mismo. Sólo refiere que se trata de unas hierbitas tomadas para contrarrestar una posible infección, manifestada con una intensa punzada y con la presencia de flujo continuo. La toma de este remedio lo relaciona directamente con la disminución de su molestia.

La misma mujer reconoce la aplicación de inyecciones sin prescripción médica ante la irregularidad de la regla, y establece una relación directa entre aquéllas y la suspensión definitiva de la menstruación.

La mayoría de las mujeres dijeron consultar habitualmente al médico. Algunas lo hicieron en relación directa con la menopausia y síntomas específicos, mientras otras acudieron por razones diferentes como intervenciones quirúrgicas, dietas, diabetes y consultas ginecológicas por otro motivo. A las que visitan al médico se les recetan pastillas cuya composición desconocen y no se preocupan por investigarlo. Sólo algunas saben que se trata de hormonas y las toman sin cuestionamiento alguno, a pesar de las molestias derivadas de su consumo, como dolor de cerebro, en el que coinciden muchas de ellas.

Aun cuando no lo hagan, mencionan al médico como la persona que visitarían en caso de contar con medios económicos.

Para estas mujeres la mente puede ejercer un control sobre la menopausia y la menstruación, de modo que sólo pensándolo se puede reglar o dejar de hacerlo; de esta manera se llega a identificar una relación causal estableciéndose una vinculación con el remedio pertinente.

De las mujeres que consultaron al médico, tres mencionaron que les recetó hormonas, dos vitaminas y dos más se refirieron a inyecciones recomendadas para:

"...no quedarse embarazada y para ir secando su matriz, para que usted no tenga trastornos...".

En los relatos se identifica una presencia importante del discurso médico, ya que incluyen con frecuencia palabras como "hormonas" o "pastillas" y construyen una explicación de carácter organicista, identificando en el cuerpo las causas de los malestares asociados con la menopausia. (Algunas la atribuyen a la alimentación, consumo de tabaco, café o alcohol, así como a la presencia de otras enfermedades como la diabetes).

Cabe resaltar que una mujer se refirió a los malos servicios del centro de salud en los siguientes términos:

“...pues muchas veces no hay (médicos), (ellos) no tienen la misma curiosidad de decirte la verdad, lo que hay...".

\section{Discusión}

En términos generales, se encontró una concepción diferencial alrededor de la menopausia entre las mujeres entrevistadas; no todas ellas la cursan con una sintomatología particular; en la frecuencia y gravedad de este cuadro influye de manera importante el significado relacionado con la menstruación, el significado del rol social de la mujer, la forma en que se concibe el envejecimiento, la fuente de información, la actitud, la comunicación y el concepto de la pareja.

La identidad femenina, al igual que la masculina, se construye socialmente y en este proceso se define también la relación entre hombres y mujeres, fundada básicamente en el ejercicio del poder de aquéllos sobre éstas. Esta dinámica de poder se puede reproducir también en el contexto de los servicios de salud. Las explicaciones que las mujeres nos ofrecieron en relación con la menopausia reflejaban en parte el punto de vista de sus médicos, y quiere decir que la información proporcionada por estos profesionales se asume como única y verdadera, pues esta figura tiene un poder legitimado socialmente; en los discursos se encuentra además una descripción de la persona vista como una víctima (es algo que como mujeres nos toca vivir), ignorante de las causas, en algunos casos dependiente de la medicina tanto para el diagnóstico como para el tratamiento, reflejo de algunas de las características sociales de la identidad femenina: el sufrimiento y la dependencia, señaladas por autoras como Szasz. ${ }^{21}$

Tres son las acciones claramente identificadas de estas mujeres: ausencia de una práctica concreta, toma de remedios caseros y visitas al médico, si bien es cierto que ninguna de ellas es excluyente, los discursos expresados y la frecuencia de las mismas (la toma de remedios casi está ausente), sugieren una concepción más común de la menopausia como una enferme- 
dad de competencia única de la medicina, y en este sentido resulta inapropiado cualquier esfuerzo, teórico o práctico, para enfrentarlo. ${ }^{22}$

La consulta médica, cuya función en este caso se encamina a la legitimación de la menopausia concebida como una enfermedad, hace posible su medicalización. Este reconocimiento legitima los síntomas de las mujeres (molestias y comportamientos) ante ellas mismas y ante la sociedad, idea expresada en otros estudios. ${ }^{22}$

Algunas de quienes acudieron al médico dijeron desconocer el tipo de pastillas recomendadas; esto no sólo da cuenta de una actitud pasiva hacia el evento, sino que es una manera de sumisión ante una autoridad reconocida, en este caso representada por la medicina.

Las políticas públicas, en relación con la mujer y los servicios de salud, han estado orientados de forma preferente al control de la natalidad ${ }^{23}$ sobre otros aspectos de la vida de las mujeres, como la menopausia.

La vivencia de la menopausia no depende únicamente de las características del propio evento, si no que se ve alimentada por otras representaciones como la experiencia más amplia del ser mujer, en donde el ejercicio de la capacidad reproductiva adquiere un peso importante; la concepción del cuerpo, manifestada mediante mitos; lo que significan la sexualidad, la menstruación, la fertilidad, la vejez; construcciones todas ellas formadas socialmente y en tanto que éste es su carácter, han permeado la identidad de las mujeres.

En este sentido se observa que la sintomatología mencionada, y sobre todo la experiencia de significados asociada al proceso de la menopausia, revela el papel jugado por la noción del ser mujer, que en nuestra sociedad destaca características muchas veces representativas de una desventaja de las mujeres frente a los hombres, ${ }^{21}$ entre ellas lo que se valora, reconoce y muchas veces se impone a la mujer es el ejercicio de su capacidad reproductiva. Se puede decir que de esto se desprende una vivencia casi trágica de la menopausia, pues está directamente relacionada con el cese de la fertilidad, resquebrajando la identidad femenina, construida sobre estas ideas.

En este estudio no se identificaron representaciones "puras", quizá porque su propio carácter las mezcla constantemente; sin embargo se pueden señalar algunos atributos que por la frecuencia de referencia pudieran definirla. Concebida como proceso natural o no, la menopausia representa el término de la vida fértil de las mujeres, el paso hacia el envejecimiento, la liberación de temores y molestias, entendiendo por ello embarazo y menstruación, respectivamente.

Uno de los elementos constitutivos de las representaciones sociales son los conocimientos que las personas tienen sobre el objeto de la representación. En el caso de nuestras entrevistadas la vía oral resultó ser la fuente principal de transmisión de conocimientos. Se habla aquí de un conocimiento empírico, reforzado por la propia experiencia de las mujeres.

Si bien es cierto que las representaciones sociales se alimentan y van cambiando a partir del contacto con nuevos ámbitos de experiencia y conocimiento, los ritmos de transformación no siempre se corresponden. En nuestra cultura prevalece una idea sobre el significado de ser mujer, que ejerce una influencia importante sobre la satisfacción y realización que ellas expresan sobre su vida.

Por otro lado, cuanto la información nos deja ver es que la incorporación de las mujeres al proceso productivo no ha implicado necesariamente un cambio en la representación social que de ella se tiene: habría que explorar el efecto tenido por esta incorporación en distintos sectores laborales. Sin embargo, algunas de nuestras informantes se dedicaban al comercio, pero la condición de trabajar fuera de casa no evidenció concepciones diferentes.

El uso de servicios de salud no es independiente de los significados que las personas construyen; todo lo contrario, en tanto que práctica social es dependiente de ellos; por esta razón se resalta aquí la importancia de explorar ese campo simbólico-representacional como una aproximación al entendimiento del comportamiento social. Las mujeres de este estudio han integrado el discurso médico en su representación de la menopausia; esto actúa en dos sentidos: hace que las mujeres usen los servicios de salud por este motivo y, a su vez, su uso refuerza la representación que se tiene de ellas.

Que las mujeres acudan a consulta puede interpretarse como un logro desde el punto de vista institucional, no obstante, una vez que las mujeres van ¿qué información se les proporciona?, ¿qué ideas acerca de la menopausia se les transmite?, ¿qué papel juega el personal médico en la solución de sus demandas y necesidades?, ¿qué opciones de tratamiento se les ofrece?, ¿de qué manera puede mejorarse este servicio? Son interrogantes abiertos para la reflexión, en la cual valdría la pena contemplar aquellas aproximaciones que nos acerquen cada vez más a los significados que construyen los actores sociales. Lo anterior debe llamar la atención sobre las ventajas derivadas de la exploración de la vivencia que las mujeres, y 
cualquier otro sector de la población, tienen con relación a eventos que han preocupado a la medicina y a los servicios de salud. De esta manera, al incorporar este conocimiento al ejercicio médico profesional, los signos y síntomas se interpretarán contextualmente y no sólo como algo orgánico, esto puede mejorar sin duda la atención y ampliar las posibilidades prescriptivas más allá de la mera medicalización.

Las transformaciones de las representaciones sociales no son responsabilidad única del sector salud, pero como autoridad legitimada su acción puede jugar un papel muy importante en la desmitificación de ideas y en la conformación de otras que no subordinen la identidad femenina.

\section{Referencias}

1. Billig, M, C ondor S, Edwards D, G ane M, Middleton D, RadleyA. Ideological dilemmas: A social psychology of everyday thinking. Londres: Sage, 1988.

2. Edwards D. D iscourse and Cognition. Londres: Sage, 1996.

3. Fernández P. Teorías de las emociones y teoría de la afectividad colectiva. Iztapalapa 1994;14:89-112.

4.W ittgenstein L. Investigaciones filosóficas. Barcelona: Crítica, 1953. 5. Jodelet D. La representación social: fenómenos, concepto y teoría. En: Moscovici S, ed. Psicología So cial II. Pensamiento y vida social. Psicología social y problemas sociales. Barcelona: Paidós, 1984:469-494.

6. Ibáñez T, ed. Ideo logías de la vida cotidiana. Barcelona: Sendai, 1988.

7. Jones J. Embodied meaning: Menopause and the change of life. Soc W ork Health Care 1994;19:43-65.

8. MacPherson KI. Menopause as disease: The social construction of a metaphor.AN SAdv N urs Sci 1981;3:95-113.
9. IbáñezT. Representaciones sociales:Teoría y Método. En:Psicología social construccionista. Guadalajara, México: Universidad de Guadalajara, 1994:153-216

10. Shotter J. Action, joint action and intentionality. En: Brenner M, ed. The structure of action. 0 xford: Blackwell, 1980:19-43.

11. Gadamer HG. Verdad y Método II. Salamanca: Ediciones Sígueme, 1992:130

12. Lolas SF. Las ciencias sociales como discurso de la salud reproductiva. El ejemplo del climaterio femenino. Cad Saude Publica 1998;14:131-134. 13. Sennott-Miller L. La situación de salud y socioeconómica de las mujeres de edad mediana y avanzada en América Latina y el Caribe. En: 0 rganización Panamericana de la Salud/A sociación A mericana de personas jubiladas. Las mujeres de edad mediana y avanzada en América Latina y el Caribe.W ashington, DC :: O PS/AARP, 1990.

14. Rosenthal MB. Psychological aspects of menopause. Prim Care 1979;6:357-364.

15. Chirawatkul S, Manderson L. Perceptions of menopause in northeast Thailand: Contested meaning and practice. Soc Sci Med 1994;39: 1545-1554.

16. Scrimshaw S, Hurtado E. Rapid assessment procedures. Los Angeles: The Regents of the University of $\mathrm{C}$ alifornia/The United $\mathrm{N}$ ations U niversity, 1988.

17. Salamonovitz A. Del silencio a la palabra. Reflexiones psicoanalíticas sobre la depresión. México, D.F.: Círculo Psicoanalítico Mexicano, 1999.

18. G lasser BG, Strauss A.The D iscovery of G rounded Theory: Strategies for Q ualitative Research. C hicago:Aldine, 1967.

19. Strauss A, C orbin J. Basics of $Q$ ualitative Research: Grounded Theory Procedures and Technics. N ewbury Park: Sage, 1990.

20. Beauvoir S. La vejez. México, D.F.: Hermes, 1980

21. Szasz I. Género y Salud. Propuestas para el análisis de una relación compleja. En: Bronfman, M y Castro, R coords. Salud, Cambio social y Política. Perspectivas desde América Latina. México, D.F.: EDAMEX, 1999:109-121.

22. King $C h R$. Parallels between neurasthenia and premenstrual syndrome. Women \& Health 1989;15:501-513

23. 0 rganización Mundial de la Salud. Investigaciones so bre la menopausia en los años noventa. Serie de informes Técnicos 866. España: O MS, 1996. 